\title{
Lumbosacral evoked potentials and vesicourethral function in patients with chronic suprasacral spinal cord injury
}

\author{
M G Lucas, D G Thomas
}

\begin{abstract}
Persistent detrusor acontractility despite normal somatic reflex activity in some patients with high spinal cord injury is an enigma. Previous work has suggested disordered integration of afferent activity in sacral roots or the sacral spinal cord. Forty male patients with chronic stable suprasacral cord lesions were studied by filling and voiding videocystometrography, and recording lumbosacral evoked potentials from posterior tibial nerve stimulation. Only five of 15 patients with decreased detrusor contractility had abnormal lumbosacral evoked potentials. Similar abnormalities were found in four of 11 patients with efficient hyperreflexic bladders. The finding of normal lumbosacral evoked potentials in the majority of patients with suprasacral cord injuries and decreased detrusor contractility supports the argument that the pathophysiology of this specific form of neurogenic bladder dysfunction is multifactorial.
\end{abstract}

Patients with suprasacral spinal cord lesions usually develop a hyperreflexic type of bladder function with varying degrees of detrusor sphincter dyssynergia (DSD). There is, however, a group of such patients in whom there is normal return of somatic reflex activity, but where the bladder remains acontractile, or there is considerable delay in return of reflex activity. Abnormalities of component parts of the lumbosacral evoked potentials (LSEP) have been shown in such patients ${ }^{1-3}$ suggesting that there is an occult dysfunction of sensory/ motor integration at the sacral cord level. Such abnormalities have also been shown in cord injured patients in whom transurethral sphincterotomy has failed to improve bladder emptying because of poorly sustained detrusor contractions. ${ }^{4}$

Evoked potentials recorded from the spinal cord below a traumatic injury demonstrate afferent neuronal activity in the isolated cord segment. ${ }^{5}$ This may be particularly relevant to the behaviour of the isolated nerve pathways associated with micturition. Past techniques of recording evoked potentials have included both invasive recording from the intrathecal space ${ }^{67}$ and from the epidural space. ${ }^{8}$ The use of epidural space electrodes for monitoring spinal cord potentials during scoliosis surgery is now popular. ${ }^{9}$
Others who feel that such invasive recording techniques are unjustifiable and unethical, have concentrated on refining methods of detecting spinal cord potentials using skin surface electrodes. Many different details of technique have been employed ${ }^{10-13}$ and these account for considerable discrepancies between the results reported from various centres. All lumbosacral recordings, however, have detected a primarily negative, travelling, potential overlying the sacral roots, the " $R$ " wave and a larger amplitude response with a decreased conduction rate over the conus medullaris, the " $S$ " wave (fig 1). There is agreement that the " $R$ " wave probably represents ascending afferent root potentials, and the " $S$ " wave represents recruited potentials in the afferent interneuronal structures of the conus medullaris. ${ }^{11114}$ The precise significance of the " $A$ " wave and the "P" wave remains uncertain. ${ }^{15}$

LSEPs are complex and difficult neurophysiological recordings to perform and have only previously been reported in relation to bladder function, from one centre. ${ }^{1-4}$ These studies have shown that there is a correlation between the presence of abnormal LSEPs and detrusor hypocontractility in patients with high spinal cord injury, thus implying that the "abnormal" bladder function in these patients is due to disorder integration of sacral afferent activity. The aim of this study was to see whether these data are reproducible in a similar population of patients with cord injuries.

\section{Patients and method}

Forty patients with chronic stable suprasacral spinal cord lesions had a combination of LSEP

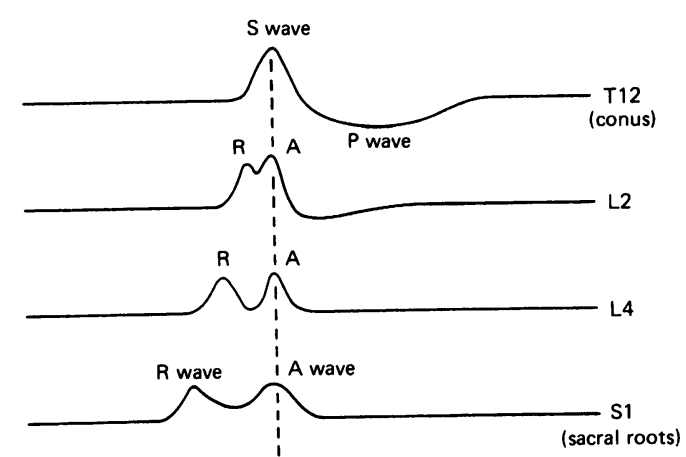

Figure 1 Diagrammatic representation of a typical lumbosacral evoked potential from stimulation of the posterior tibial nerve at the knee. The diagram shows traces obtained at four anatomical levels, and features the most significant waveforms observed at those levels. The $A$ wave may represent volume conduction of the $S$ wave. 
measurements and full "videourodynamic assessment" of vesico urethral function. All patients were male, ranging in age from 16 to 61 years, with a median age of 24 years. The range of duration of the injury was from six to 444 months, with a median duration of 38 months. There were 13 cervical cord injuries, 10 injuries to the upper thoracic spinal cord (T6 and above), 13 thoracic spinal cord injuries at T7 to T9 and four injuries at T11 or below. All except the three lowest cord lesions had complete loss of motor function below the lesion. These same three patients, plus one at $\mathrm{C} 7$ and one at $\mathrm{T} 9$, had minimal sensory sparing.

Twenty two normal volunteers, who were mostly Health Service workers, also had LSEP recording. They were nine females and 14 males, age range 19 years to 46 years, median age 26 years.

\section{LSEP recording}

Subjects lay supine on a bed in a warm room with as little noise and direct lighting as possible, and were encouraged to relax completely, and preferably go to sleep. Sedation was not used. Each study took approximately $45 \mathrm{~min}$ utes of preparation and 45 minutes of recording. No subject complained of pain.

Stimulation electrodes (Medtronics 3795) were applied to the skin of the popliteal fossa (cathode) and suprapatellar region (anode) on both legs. To stimulate both legs simultaneously the electrodes were connected in parallel. Unequal limb resistances were balanced out by means of a potentiometric device operating in much the same way as the balance control of a domestic "hifi" system. Thus an equal and synchronous stimulus could be applied to both legs. A grounding electrode was applied to each thigh just above the knee.

Recording electrodes (Beckmann $1.5 \mathrm{~cm}$ diameter recessed electrodes Sensormedics 650951) were applied to the skin over the spinous processes of $\mathrm{T} 12, \mathrm{~L} 2, \mathrm{~L} 4$ and $\mathrm{S} 1$ vertebrae. A similar electrode was placed at T6 as a common reference site.

Square wave stimuli of $0.2 \mathrm{~ms}$ duration and variable current were applied to the posterior tibial nerve at the knee, each one during the quiet phase of the electrocardiogram. The stimulus level was controlled by monitoring the ipsilateral electromyographic response of the triceps surae muscle, initially for maximum " $H$ " reflex response (a spinal reflex which occurs above the afferent nerve threshold), and then for the maximum " $M$ " response (due to direct motor stimulation of the muscle) in each case. Recordings were made with unilateral stimulation at maximum " $\mathrm{H}$ " and maximum " $M$ " level, and then with simultaneous bilateral leg stimulation at both maximum " $H$ " and maximum " $M$ " response levels. ${ }^{16}$ One hundred stimuli were applied for each recording sequence, and the responses were averaged. Each sequence was repeated at least twice to check reproducibility.

The equipment used as the basis of the system was the Dantec Neuromatic $2000 \mathrm{M}$, with no intrinsic alterations. The amplifier bandwidth employed was from $50 \mathrm{hz}$ to $2 \mathrm{khz}$.
The equipment had additional switching circuitry to enable any two of up to eight input channels to be viewed at a time.

Thus evoked potentials from T12, L2, L4 and $S 1$, all referenced to a single electrode at T6, were recorded for each train of stimuli.

The parameters measured in each investigation were as follows: at $S 1$, the " $R$ " wave latency and amplitude, and the peak to peak latency of " $R$ " to " $A$ " waves were measured (fig 1). At T12 the latency and amplitude of the "S" wave and the peak to peak " $S$ " wave to " $P$ " wave were measured.

\section{Videourodynamics}

Combined filling and voiding cystometrography, with simultaneous radiological screening and video recording were used to assess vesicourethral function in detail and in accordance with the recommendations of the International Continence Society. Our usual technique for studying neurogenic bladder dysfunction was used. ${ }^{17}$ This involved concurrent measurement of intravesical and intrarectal pressures using fluid filled $3 \mathrm{Fr}$. "Vygon" catheters attached to "Statham" strain gauge transducers. Bladder filling was via a second $3 \mathrm{Fr}$ catheter at $<20 \mathrm{mls}$ per minute, filling on top of pre-existing residual urines. Electronic derivation of detrusor pressure and print out of pressure recordings were carried out using a "Disa" 2100 Urodynamic System.

Bladder function was categorised, for the purposes of this study as 1) hyperreflexic with efficient emptying (residual urine less than $100 \mathrm{mls}$ ), 2) hyperreflexic with poor bladder emptying due to DSD, 3) hyperreflexic with poor emptying due to unsustained detrusor contractions, and 4) acontractile.
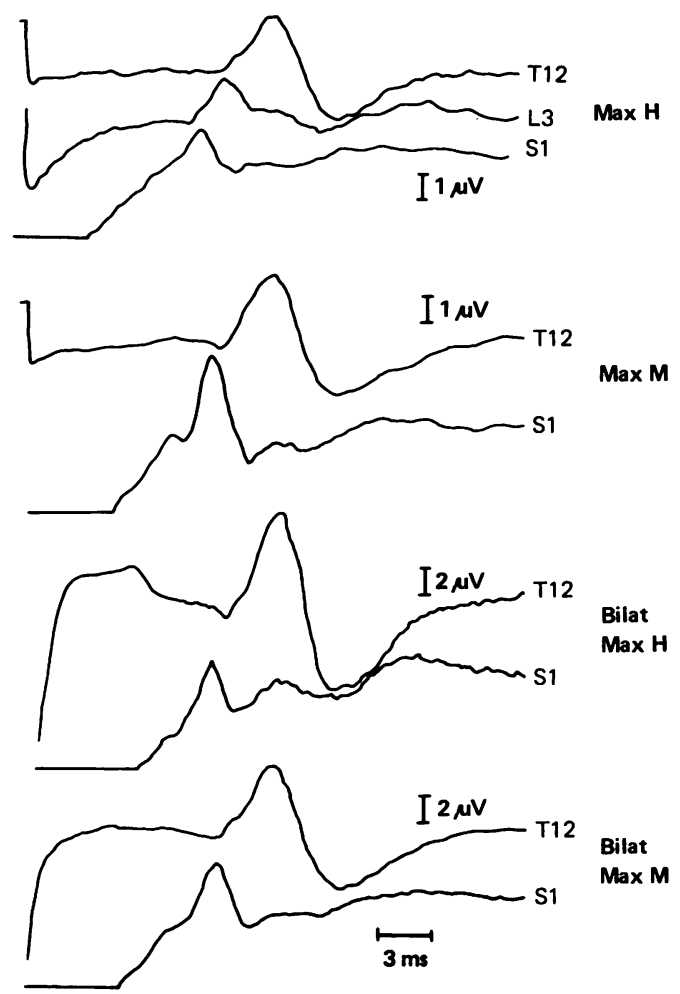

Figure 2 LSEP recorded from a normal subject. The average recordings are shown resulting from repeated stimulation at four different levels of intensity. 
Table 1 Comparison of the mean amplitudes and latencies (corrected for height) for normal subjects $(n+22)$ and those cord injured patients in whom recordings were present, and considered normal $(n+23)$. Recordings at maximum " $M$ " stimulation

\begin{tabular}{lllll}
\hline & $\begin{array}{l}\text { Corrected " } R \text { " } \\
\text { wave } \\
\text { latency ms }\end{array}$ & $\begin{array}{l}\text { Amplitude of " } R \text { " } \\
\text { wave } \\
u \text { volts }\end{array}$ & $\begin{array}{l}\text { Corrected " } S \text { " } \\
\text { wave } \\
\text { latency } m s\end{array}$ & $\begin{array}{l}\text { Amplitude of " } S \text { " } \\
\text { wave } \\
\text { uvolts }\end{array}$ \\
\hline $\begin{array}{l}\text { Normal subjects } \\
\text { Spinal injured group }\end{array}$ & $\begin{array}{l}10 \cdot 75+0 \cdot 49 \\
10 \cdot 86+0 \cdot 7\end{array}$ & $\begin{array}{l}2 \cdot 99+1 \cdot 22 \\
2 \cdot 77+1 \cdot 11\end{array}$ & $\begin{array}{l}14 \cdot 18+0 \cdot 59 \\
14 \cdot 47+0 \cdot 74\end{array}$ & $\begin{array}{l}4 \cdot 31+14+62 \\
4 \cdot 14+2 \cdot 03\end{array}$ \\
\hline
\end{tabular}

Table 2 LSEP responses in 40 spinal cord injured subjects

\begin{tabular}{|c|c|c|c|}
\hline Group & $\begin{array}{l}\text { Number of } \\
\text { patients }\end{array}$ & $\begin{array}{l}\text { Percentage of } \\
\text { total }\end{array}$ & Comment \\
\hline $\begin{array}{l}\text { A All responses present } \\
\text { latencies normal }\end{array}$ & 23 & 57.5 & \multirow{5}{*}{$\begin{array}{l}S \text { wave absent in all } \\
R \text { wave absent in all } \\
1 \text { delayed } S \text { wave } \\
1 \text { delayed } R+S \text { wave } \\
1 \text { missing } S \text { wave } \\
1 \text { missing } R \text { wave } \\
\text { see text } \\
\text { see text }\end{array}$} \\
\hline$B$ " $S$ " or " $R$ " wave missing & 4 & 10 & \\
\hline$C$ “" $S$ " or " $R$ " wave delayed & 2 & 5 & \\
\hline D One missing, one delayed & 2 & 5 & \\
\hline $\begin{array}{l}\text { E No detectable responses } \\
\text { F Responses present but not } \\
\text { reliably reproducible }\end{array}$ & $\begin{array}{l}3 \\
6\end{array}$ & $15^{7 \cdot 5}$ & \\
\hline
\end{tabular}

Table 3 Bladder function in different LSEP groups

\begin{tabular}{lccccc}
\hline & $\begin{array}{l}\text { Reflex } \\
\text { efficient }\end{array}$ & $\begin{array}{l}\text { Reflex } \\
\text { DSD }\end{array}$ & $\begin{array}{l}\text { Reflex } \\
\text { PSC }\end{array}$ & Acontractile & Total \\
\hline A All responses normal & 7 & 6 & 6 & 4 & 23 \\
B "S", or "R", wave missing & & 2 & 1 & 1 & 4 \\
C "S" or "R" wave delayed & 1 & 1 & 1 & & 2 \\
D One missing, one delayed & & 2 & 1 & 2 & 2 \\
E No responses & 3 & 11 & 10 & 1 & 6 \\
F Equivocal responses & 11 & 8 & 40 \\
$\quad$ Total & & & & & \\
\hline
\end{tabular}

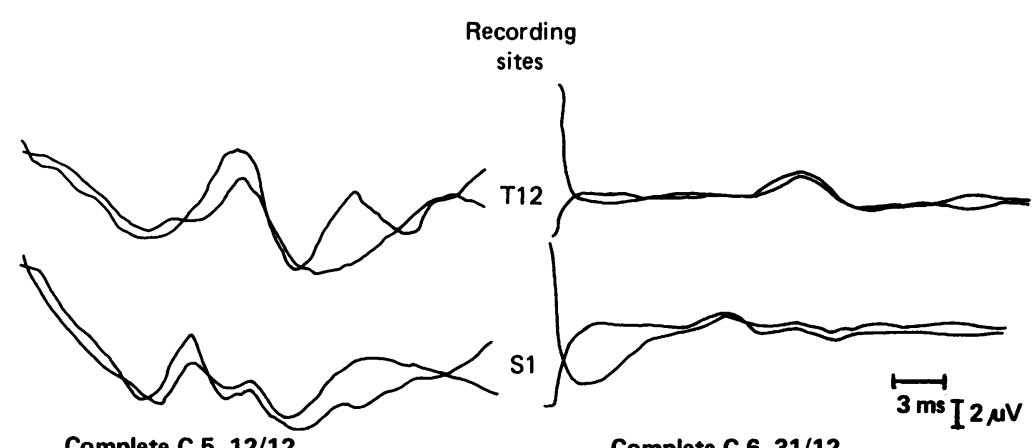

Complete C.5. 12/12

Complete C.6. 31/12
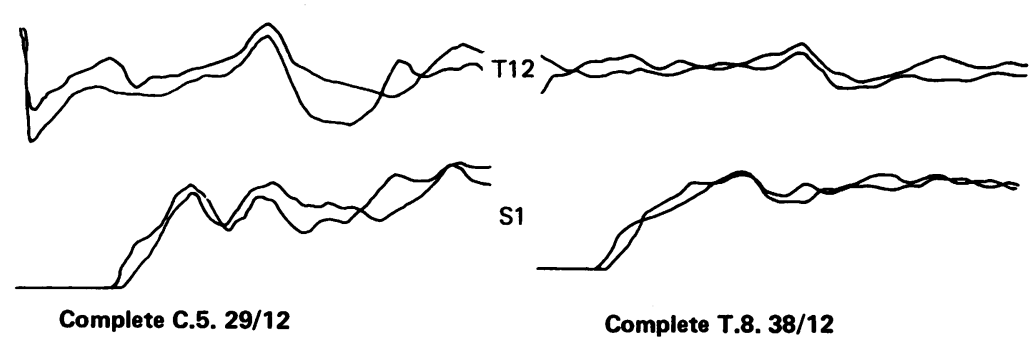

Figure 3 Normal LSEPs recorded from four patients with high spinal cord injury," showing level of injury and time since injury. All recordings made at maximum " $M$ " stimulation. All four of these patients had normal return of somatic reflexes, and a normal bulbocavernous reflex, but a persistently acontractile bladder. Original superimposition of responses using "store" facility of Dantex "Neuromatic"; then traced over for photography.
Results

A typical LSEP recording from a normal subject is illustrated in fig 2 . In all normal subjects the characteristic patterns of evoked potentials, and the mean latency times of " $R$ " waves, " $S$ " waves and " $A$ " waves, once corrected for height, were identical to those previously reported by Dimitrijevic et al, ${ }^{12}$ except that the " $S$ " to "P", peak to peak latency time was shorter. The reason for this difference is not clear.

The majority of LSEPs recorded from patients with spinal cord injuries were normal. A comparison of latency and amplitude values for both normal and cord injured patients is shown in table 1. LSEPs in the group of 40 patients were categorised according to the classification described by Lehmkuhl et $a^{16}$ (table 2).

Group E consisted of one patient with a T12 lesion, one with an L4 lesion, and one with a T5 lesion with distal cord infarction. These three patients had been included in the study to check the validity of recordings on the hypothetical grounds that significant abnormalities of lumbosacral evoked potentials would be present.

An additional group has been included for the purposes of this study, Group F. This consisted of patients for whom the investigator was able to measure responses, but these were not reliably reproducible. In no cases was the failure to record a response attributable to obesity, poor preparation, spasm or sweating and these were therefore regarded as abnormal responses. In all but one the specific abnormality was difficulty in recording the " $R$ " wave. The last patient had both unconvincing " $R$ " wave and " $S$ " wave responses.

Correlation of LSEPs with bladder function Eleven patients $(27.5 \%$ of the total) had efficiently emptying hyperreflexic bladders (that is, leaving a residual urine of $<100 \mathrm{mls}$ after a voiding sequence). Eight of these had early or minimal DSD, and one had synergic voiding. Two patients emptied adequately despite prolonged DSD with high swinging pressures, but voided effectively in between contractions. In all the patients in this group, the "S" wave was of normal latency. " $R$ " wave abnormalities were present in four patients. There were no responses completely missing in any of the patients.

Eleven patients had poorly emptying hyperreflexic bladders, due to prolonged DSD. Two of these had had previous transurethral sphincterotomy, but DSD had recurred after a few years. No neurophysiological relationship was seen in this group, however, since all LSEP groups were represented.

A further 10 patients had poorly sustained hyperreflexic bladder contractions leading to inefficient emptying (table 3). Six of these 10 patients had normal LSEPs (group A), but groups B, C, D and E were also represented. Five of the 10 had undergone previous sphincterotomy and had poorly sustained ineffective contractions. These five patients, however, still 
The final group of eight patients had noncontractile bladders (table 3). Of these, five had persistent acontractility for between 13 and 37 months (median 32 months) after injury, in the presence of a high suprasacral cord lesion and return of somatic reflex activity, that is, somatic hyperreflexia below the level of the neurological lesion and both the glans, bulbar and anal skin reflexes present on clinical examination. Four of these five patients had entirely normal LSEPs (fig 3), and the fifth had abnormal " $R$ " waves. One patient with a T12 lesion and partial motor recovery in one leg (Grade 2) had no contractile activity of his bladder at six months, and LSEPs showed absent " $S$ " waves with normal " $R$ " waves. The two remaining patients had served as controls, since there was no clinical evidence of any sacral reflex activity in either of these patients.

\section{Discussion}

The results of this study contrast with work previously reported. ${ }^{1-4}$

Light et al $^{1}$ studied 13 patients with a mixture of delayed return of bladder reflex activity, and persistent acontractility. All had high spinal cord lesions. Nine of the patients had abnormal LSEPs, while in four they were not carried out. Later, ${ }^{2} 36$ patients were reported with high spinal cord injury, compared with a similar group of age matched controls. Fifteen of the 36 patients had an abnormal " $S$ " wave of LSEP and all of these had "abnormalities of bladder function". Only three patients out of the remaining 21 with normal LSEP had "abnormal bladder function". Beric et $a l^{\beta}$ reported presumably the same group of 15 patients selected from 130 patients with spinal cord injury who had undergone LSEP testing. The 15 had no evidence of a second bony injury radiologically, and all had some other discrete neurophysiological findings suggesting localised lumbosacral cord dysfunction. The particular abnormalities described in these studies include acontractility, hypocontractility following transurethral sphincterotomy, and a slow "rise time" of detrusor pressure to maximum contraction.

A previous study ${ }^{4}$ reviewed a group of nine patients with high spinal cord injury who had had a poor functional result from transurethal sphincterotomy due to poor detrusor contractility. Eight of these nine had abnormal LSEP (" $R$ " and " $S$ " wave abnormalities) and one had evidence of a lower motor neuron lesion. It has been proposed that a combination of abnormal LSEP and a slow rise time to maximum detrusor contraction should be a contraindication to bladder outlet surgery in patients with suprasacral lesions. ${ }^{4}$

These previous findings ${ }^{1-4}$ suggest that in a number of cord injured patients with delayed or absent bladder activity there is a profound defect of afferent conduction through either sacral roots or the sacral cord. However, only one such case was demonstrated in the current study. Four of the six patients in this series with delayed or absent return of bladder reflex activity, who also have high suprasacral cord lesions with normal somatic and conus reflexes, had entirely normal LSEPs.

The patients in this study were perhaps not strictly comparable to those in the study from Houston, Texas. ${ }^{3}$ Our study included 10 patients with lesions below T8, whilst the Houston series included only T8 lesions and above. In this study, however, the important finding was that the majority of patients with detrusor hypocontractility had normal LSEPs. Of these 15 patients, eight were cervical injuries, six were paraplegic at T8 or above and only one at T9. Thus the comparison of these studies is valid.

No clear correlation could be shown between bladder function and the LSEP findings. A similar study of patients with acute cord injuries failed to show any relationship between sacral reflex latency studies and the patterns of bladder function which develop after cord injury. ${ }^{18}$

Afferent activity from the bladder and proximal urethra clearly plays a vital role in the patterns of bladder behaviour which develop following spinal cord injury. An anal stretch during voiding will often totally eliminate dyssynergic sphincter contractions and allow more effective bladder emptying. ${ }^{19}$ Similarly, electrical stimulation of the pudendal nerves in patients with neurogenic bladder dysfunction and synergic voiding may result in improved bladder emptying, whilst similar stimulation in patients with DSD may considerably worsen the dyssynergia and prevent voiding. ${ }^{20}$ These findings related to afferent input affecting detrusor activity lend support to a "gate" theory of integration of afferent and efferent activity at sacral cord level. ${ }^{21}$

The behaviour of the cord injured bladder remains largely an enigma. Combined pharmacological and morphological studies of the bladder muscle of spinal cord injured patients would reveal more about the factors controlling detrusor activity at neuromuscular level. Complex neurophysiological tests will become, increasingly, a part of the investigation and rehabilitation of patients with cord injuries and specific tests may evolve to examine the integration of sensory and motor activity of the bladder.

The results of this study, however, do not contradict previous work but provide additional data on this subject, and emphasise that functional abnormalities of the urinary storage/voiding system can rarely be explained on the basis of just one pathological lesion.

We thank Drs $M$ Dimitrijevic and $A$ Sherwood, Department of Clinical Neurophysiology and Rehabilitation, Baylor College of Medicine, Houston, Texas, and members of their department for teaching us the technical aspects of lumbosacral evoked potential recording. Mr Philip Wild and Mr Graham Harston of the department of Medical Physics and Clinical Engineering, Lodge Moor Hospital, Sheffield, were responsible for creating the auxiliary equipment required for this work. The equipment was partially funded by the Swann Morton Trust. 
1 Light JK, Fanagel J, Beric A. Detrusor Areflexia in suprasacral spinal cord injuries. $J$ Urol $1985 ; 134: 295-7$.

2 Light JK, Beric A, Eppel SM. Evidence for a sensory abnormality and bladder dysfunction in high spinal cord injury. Proc Int Continence Soc. Neurourology and Urodynamics 1987;6:230.

3 Beric A, Dimitrijevic MR, Light JK. A clinical syndrome of rostral and caudal spinal injury; neurological, neurophysiological and urodynamic evidence for occult sacral lesion. J Neurol Neurosurg Psychiatry 1987;50:600-6.

4 Light JK, Beric A, Wise PG. Predictive criteria for failed sphincterotomy in spinal cord injury. $J$ Urol 1987;138: 1201-4.

5 Sedgewick EM, El Negamy E, Frankel H. Spinal cord potentials in traumatic paraplegia and quadriplegia. $J$ potentials in traumatic paraplegia and quadr
Neurol Neurosurg Psychiatry 1980;43:823-30.

6 Magladery JW, Porter WE, Park AM, Teasdall HD. Electrophysiological studies of nerve and reflex activity in normal man. iv the two neurone reflex and identification etc. Bull Johns Hopkins Hospital 1951;88:499-519.

7 Ertekin C. Evoked electrospinogram in spinal cord and peripheral nerve disorders. Acta Neurol Scand 1978; 57:329-44.

8 Shimoji K, Matsuki M, Shimizu H. Waveform characteristics and spatial distribution of evoked spinal electrogram in man. $J$ Neurosurg 1977;46:304-13.

9 Jones SJ, Edgar MA, Ransford AO, Thomas NP. A system for the electrophysiological monitoring of the spinal cord during operations for scoliosis. J Bone Joint Surg (Br) 1983;65:134-9.

10 Cracco RQ. Spinal Evoked Response: Peripheral nerve stimulation in man. Electroencephalogr Clin Neurophysiol 1973;35:379-86.

11 Schiff JA, Cracco RQ, Rossini PM, Cracco JB. Spine and scalp somatosensory evoked potentials in normal subjects and patients with spinal cord disease. Electroencephalogr
Clin Neurophysiol 1984;59:374-87.

12 Dimitrijevic MR, Lehmkuhl LD, Sedgewick EM, Sherwood AM, McKay WB. Characteristics of spinal cord evoked responses in man. Applied Neurophysiology (Basel) 1980;43:118-27.

13 Phillips LH, Daube JR. Lumbosacral spinal evoked potentials in humans. Neurology 1980;30:1175-83.

14 Dimitrijevic MR, Larson LE, Lehmkuhl D, Sherwood A Evoked spinal cord and nerve root potentials in humans using a non invasive recording technique. Electroencephalogr Clin Neurophysiol 1978;45:331-40.

15 Lehmkuhl D, Dimitrijevic MR, Renouf F. Electrophysiological characteristics of lumbosacral evoked potentials in patients with established spinal cord injury. Electroencephalogr Clin Neurophysiol 1984;59:142-55.

16 Delbeke J, McComas AJ, Kopec SJ. Analysis of evoked lumbosacral potentials in man. $J$ Neurol Neurosurg lumbosacral potentials in

17 Thomas DG. Clinical urodynamics in Neurogenic Bladder Dysfunction. Urological Clinics of North America 1979; 6:237-53.

18 Lucas MG, Thomas DG. Lack of relationship of conus reflexes to bladder recovery following spinal cord injury. Br J Urol 1989;63:24-7.

$19 \mathrm{Wu} \mathrm{Y,} \mathrm{Nanninga} \mathrm{JB,} \mathrm{Hamilton} \mathrm{BB.} \mathrm{Inhibition} \mathrm{of} \mathrm{the}$ external urethral sphincter and sacral reflex by anal stretch in spinal cord injured patients. Arch Phys Med Rehab 1886;67:135-7.

20 Dyro FM, Yalla SV. Refractoriness of urethral striated sphincter during voiding: studies with afferent pudendal reflex arc stimulation in male subjects. J Urol 1986; 135:732-6.

21 De Groat WC, Booth AM. Inhibition and facilitation in parasympathetic ganglia of the urinary bladder. Federatparasympathetic ganglia of the 